IX.

\title{
Bericht über die Verhandlungen der otologischen Section auf der 62. Versammlung Deutscher Naturforscher und Aerzte in Heidelberg.
}

\author{
Von \\ Dr. Sigismund Szenes \\ in Budapest.
}

I. Sitzung am 19. September $9 \mathrm{Uhr}$.

Vorsitzender: Prof. Moos (Heidelberg).

1. Prof. $\mathrm{Kuhn}$ (Strassburg), a) Ueber Otitis diabetica (der Vortrag findet sich in diesem Heft abgedruckt).

Discussion. Prof. Walb (Bonn) erwähnt einen Fall, wo er die Aufmeisselung nach 8 tägigem Bestande der Otorrhoe vornahm, und schon nach einigen Meisselschlägen stiess er anf eine grosse Zerstörung. Die periostalen Abscesse scheinen dem Redner verdäehtig zu sein und er würde in jedem Falle gleich aufmeisseln; er hält die Ôhreiterung nur für symptomatisch und glaubt eine Knochenerkrankung von vornherein annehmen zu miissen, denn anders ist es kaum möglich die grosse Destruction am Knochen zu erklären.

Prof. $\mathrm{Kub}$ n stimmt dem Vorredner bei und glanbt es mit einer acuten Caries des Knochens zu thun zu haben.

Körner (Frankfurt) erwähnt einen Fall, in welchem er friihzeitig operirte; der Eingriff verursachte zwar zunächst eine Vermehrung des Zuckers im Harn (von 0,3 Proc. anf 2,3 Proc.), doch am nächsten Tag war der Procentsatz der fribere.

Wolf (Frankfurt) möchte nicht in jedem Falle von Otorrhoe bei Diabetikern den Warzenfortsatz aufmeisseln, da das doch kein indifferenter Eingriff sei.

Prof. Kuhn betont nochmals die Indication für die frühzeitige Eröffnung des Warzenfortsatzes bei Diabetikern.

Prof. Moos (Heidelberg) glaubt auch, unter allen Umständen operiren zu mïssen.

b) Bacteriologisches bei otitis media.

Prof. Kahn berichtet iuber eine Arbeit, die ans seinem Institute stammt und sich auf die bacteriologische Untersuchung des Secretes in 23 Fallen bezieht. In 10 Fällen handelte es sich um eine acute Otitis media purulenta, bei der die Paracentese gemacht war; in 
2 Fällen war die Perioration bereits vorhanden, 7 mal hatte sich za der acuten Otitis eine Affection des Warzenfortsalzes gesellt, in 2 Fällen bestand eine chronische Mittelohreiterung und schliesslich in 2 anderen Fallen eine solche mit Cholesteatom complicirt. Das Resultat der Untersuchung war eigentlich ein negatives, insofern als atiologisch kein einheitliches Bild nachgewiesen werden konnte. Die verschiedenen Mikroben, die in den einzelnen Fallen gefunden wurden, erwähnte Vortragender von Fall zu Fall und beschrieb anch eingehend den Verlauf der einzelnen Processe.

2. Prof. Moos (Heidelberg), Histologie und Bacterjologie dex diphtheritischen Mittelohrekrankungen.

Vortragender berichtet iber die Befunde an 6 einschlägigen Fälen; in sämmtlichen war das Trommelfell nicht perforirt.

\section{I. Sitzung am 19. September 1 Uhr.}

Vorsitzender: Prof. Kuhn (Strassburg).

1. Wolf (Frankfurt), Hörprüungsworte und ihr differentiell diagnostischer Werth.

Vortragender ist iberzeugt, dass die mensehliche Sprache bei Weitem das beste Hörprufungsmittel, weil das vollkommenste, sei. Dieselbe sei von den Collegen bisher wohl quantitativ verwerthet worden, d. h. zur Prüfung der $\mathrm{Hör} \mathbf{r}$ eite, aber noch nicht genügend qualitativ, nämlich für Prüfung der Hörbreite. Der Grund dafür sei wohl darin zu suchen, dass bisher Niemand den Versuch gemacht habe, đie zur Hörprifung geeigneten Laute und Worte ihrem akustischen Werthe nach auszuwäblen und somit den complicirten Prufungsapparat der mensehlichen Sprache wesentlich zu vereinfachen. Es muissten nur solche Worte benutzt werden, in welchen der zu priffende, vom Vortragenden als selbsttönenả bezeichnete Consonant prägnant hervortritt.

Vortragender gab dann eine kurze Uebersicht der nach Tonhöhe, Klangfarbe und Tonstärke von ihm akustisch festgestellten Sprachlaute und wies nach, weshalb bei den verschiedenen Alterationen des schallznleitenden und des schallempfindenden Apparates die selbst. tönenden Consonanten je nach dem Sitz der Erkrankung gut, scbwierig oder gar nicht gehört werden, so dass sich durch diese Prüfung sämmtliche Tonlizeken nachweisen lassen. Dabei brauche man die Versuche mit der Stimmgabelreihe nicht aufzugeben, doch hätten diese den Nachtheil, dass sie weit mehr Mühe und Zeit in Anspruch nehmen und für den nicht musikalischen Kranken weit unverständlicher und angenaner seien, als die Versuche mit den Hörprüfungsworten.

Schliesslich schlägt Vortragender zur Verwendung 3 Gruppen von Worten vor: Zischlate, Explosivlaute und tiefe $R$ La ate, insbesondere das Zungenspitzen- $R$, und zeigt, welche Veränderung and Verwechselung die betreffenden Consonanten bei der Perception seitens der verschiedenen Ohrenkranken erleiden und somit Rückschluss anf den Sitz und Charakter des Leidens gestatten. 
Diseusion. Prof. Moos (Heidelberg) empfieht, chronische Falle stets mit den Stimmgabela za untersuchen.

Barth (Berlin) glaubt, dass die Hörprifungsworte ans der Reihe der Zahlen genommen werden sollen, wie dies schon Lucae langst emptohlen habe.

Hartmann (Berlin) fragt, ob Woll zur Controlle auch mit Stimmgabeln untersuche.

Wolf hat die Controlversuche gemacht, doch erwahnt er die schwache Seite der Stimmgabeluntersnchungen, wonach man uberhaupt bei einseitigen Erkrankungen nieht leicht zum Ziele komme.

Barth giebt zu, dass Stimmgabeln of nur gef it hlt und nicht gehört werden, doch kann man sich durch Quer- und Kreuzfragen stets davon uberzeugen, ob die Anssage des Patienten richtig ist.

Prof. Kessel (Jena) hält für den praktischen Arzt für iiberaus wichtig das isolirte Prufen; das Prufen soll mit den Consonanten und Vocalen einzeln vorgenommen werden. Bei der Untersuchung mit Stimmgabeln ist es angezeigt, dieselbe mit Gabeln verschiedener Tonhöhe vorzunehmen, und fand Redner stets, dass mit Zunahme der Tonhöhen die Töne der Stimmgabein undentlicher werden. Endlich kommt Redner auf die von den Patienten so oft erwähnten Ge räusche zu sprechen, deren 150 versehiedene Formen er bereits notirte, und trotzdem glaubt er nur $z w e j$ Formen annehmen $z u$ müssen, nämlich das tiefe Sieden (etwas intensiver ist das Zischen) und das hohe Klingen.

2. Privatdocent Kilian (Freiburg), Zur vergleichenden Anatomie und rergleichenden Entwicklungsgesclichte der Ohrmuskeln.

Vortragender giebt einen kurzen vorlanfigen Berieht ther seine vergleichend-anatomischen und vergleichend-entwicklungsgeschichtlichen Studien über die morphologische Bedentung der Ohrmuskeln.

Der älteste Muskel des Ohres ist nach ihm der Stapedius, es folgen der Tensor tympani und znletzt die Muskeln des ánsseren Ohres. Der Stapedius hat sich von einem Kaumuskel (dem Depressor maxillae inferioris der Amphibien, Reptilien und vieler Sauger) abgespalten. Er wird gefunden bei Eidechsenembryonen, bei Kroko* dilen, bei Vögeln and Säugern.

Der Tensor tympani, der nur bei Säugethieren vorkommt, ist gemeinsam mit dem Tensor veli palatini aus einem Theil des Musc. pterygoidens internus entstanden, also gleichfalls ans einem Ka umuskel.

Die ausseren Ohrmuskeln stammen von dem Platysma $a b$, and zwar die vor dem Ohre gelegenen von dem Gesichtstheil, die hinter dem $\mathrm{Ohr}$ gelegenen von dem dorsalen und occipitalen Theil des Platysma. Dem Platysma aber entspricht der Mylohyoideus posterior der Amphibien = Constrictor superficialis hyoideas, Pars ventralis der Haifische, weleher ein Athemmuskel ist. Die binter der Ohrmuschel gelegenen Muskeln lassen sich auf jenen Theil des Constrictor zurückfuhren, der sich hinten dorsalwärts bis zur Rückenfascie fortsetzt und die hintere Portion des Constrictor superficialis dors. bildet. 
3. Kata (Berlin), Veber die Endigangen des Nervas cochleae im Corti'sehen 0rgan mit Demonstration von Präparaten (der Vortrag ist in diesem Hefte abgedruekt).

4. Prof. Stein brügge (Giessen), Ueber das Verhalten der Roissner"schen Membran bei intrucranieller Drucksteigerung mit Demonstration dazu gehöriger Präparate.

Vortragender berichtet über eine pathologische, die Reissnersche Membran betreffende Veränderung in den beiderseitigen Gehörschnecken eines 12 jährigen Knaben, welcher an einem Tumor der Zirbeldrüse zu Grunde gegangen war. Eine wenige Tage vor dem Tode vorgenommene Functionsprïfung hatte verminderte Hörschärfe sowohl für Luft-, als auch für Knochenleitung ergeben, doch konnte dies Resultat nicht für die gefundenen labyrinthären Veränderungen maassgebend sein, da ausserdem eine rechtsseitige Otitis externa mit Trïbung und Verdickung des reehten Trommelfells bestand und der Patient sich im Uebrigen bereits in einem halb somnolenten Zustande befand. Die ophthalmoskopisehe Untersuchung hatte namentlich rechterseits beginnende Neuritis optica ergeben.

Bei der Section fand man ausser dem Tumor hochgradigen Hydrocephalus internus, Compression des Gehirns, Abflachung der Windungen, somit die Erscheinungen intracranieller Drucksteige. rung.

In beiden Schnecken zeigte sich die Reissner'sche Membran deprimirt, wodurch der Ranm des Ductus cochlearis erheblich beeinträchtigt worden war. Durch diese Depression erschien anch die Corti'sche Membran gegen die Köpfe der Corti'schen Pfeiler gedrängt, und die letzteren von oben nach abwärts gedrickt, an einzelnen Stellen förmlich geknickt. Im Uebrigen fanden sich Spuren beginnender Entzündung, kleine Blutextravasate zwischen den Nervenfasern, Ablösung der Epithelzellen des Duetus cochlearis, Untergang der zelligen Gebilde des Corti'schen Organs. -

Vortragender weist anf die Möglichkeit bin, dass die Depression der Reissner'schen Membran mit der intracraniellen Drucksteigerung in Zusammenhang gestanden habe. Es sei denkbar, dass die Drucksteigerung sich durch den Aquaeductus cochleae hindurch in die perilymplatischen Räume hinein fortpflanzen könne, während dieselbe dem Saceus endolymphaticus gegeniiber, anatomischer Verhältnisse halber, eng begrenzt sei.

Discnssion. Privatdocent Habermann (Prag) hielt die bisher von ihm gefundene Depression der Reissner'schen Membran als künstliche Bildung.

Barth (Berlin) fand bei Thieren eine Bauchung der Reissnerschen Membran nach unten und oben, oft sogar, wie es bei Mäusen zu finden ist, Schlängelungen in Fällen, wo eine pathologische Veränderung gar nicht vorauszusetzen wäre.

Katz (Berlin) fand bei Kindern häufig die von Steinbrïgge erwähnten Zustände und vermuthet auch, dass dieselben Folgen der meningitischen Zustände wären, bei denen eine vermehrte intracranielle Druckerhöhung zu Grunde liegt. 
5. Hartmann (Berlin), Deber die bei der Aufmeisselung des Warzenfortsatzes in Betracht kommenden anatomisehen Verhältnisse mit Demonstration von Präparaten.

Nachdem seiner Zeit durch die anatomischen Untersuchungen von Bezold and dem Vortragenden nachgewiesen worden sei, dass die Aufmeisselung des Warzenfortsatzes weiter nach vorn vorgenommen werden müsse, machte Hartmann Messungen bezitglich der Möglichkeit einer Verletzung des Nervus facialis und der Halbzirkellsanäle. Es wurden bei 50 trockenen Schlafenbeinen Horizontalschnitte durch die Mitte des Gehörgangs angefertigt und durch Messung die Entfernung des Facialkanales und Halbzirkelkanales bestimmt: 1. von der Spina supra meatum, 2. von einem $1 \mathrm{Cm}$. hinter der Spina gelegenen Punkte, der gewöhnlichen Stelle der Aufmeisselung. Die durchschnittliche Entfernung von der Operationsstelle zum Facialkanal betrug 22, zum Halbzirkelkanal 21,5 Mm. Der Halbzirkelkanal war 9 mal weniger als $20 \mathrm{Mm}$. von der Operationsstelle entfernt, $3 \mathrm{mal}$ 17, 2 mal 18, 4 mal $19 \mathrm{Mm}$; beim Facialkanal betrug die Entfernung 8 mal weniger als $20 \mathrm{Mm}$., 4 mal 28, $4 \mathrm{mal} 19 \mathrm{Mm}$. Es geht daraus hervor, dass schon bei einer Tiefe des Operationskanales von $17 \mathrm{Mm}$. Halbzirkel- oder Facialkanal verletzt werden kann. Dieselben Maasse kommen auch für die pathologischen Verhältnisse in Betracht, da eine Totalanstrebung des Warzenfortsatzes oder eine Verdickung der Corticalis nach aussen bei den Eiterungsprocessen ira Warzenfortsatz in der Regel nicht rorkomme.

Vortragender bespricht sodann die Frage, ob die Abmeisselung der hinteren Gehörgangswand, um in die Paukenhöhle einzudringen, ohne Gefahr der Verletzung von Halbzirkel- und Facialkanal gemacht werden könne. Die Frage wird verneint, da die beiden Kanäle, wie an den vorgelegten Präparaten gezeigt wird, häufig so weit nach aussen vorspringen, dass eine Verletzung derselben nicht vermieden werden kann.

Da der im Bereiche des Trommelfells liegende Theil der Paukenhöhle vom Gehörgang ans zugänglich ist, ist, um diesen freizulegen, die Abmeisselung der hinteren Gehörgangswand nicht erforderlich. Nur der obere Theil der Paukenhöhle ist den Eingriffen vom Gehörgang aus nicht immer zugänglich. Um diesen freizulegen, kann auf dreierlei Weise verfahren werden: 1. durch Abtragung der Pars ossea des Trommelfells vom Gehörgang aus vermittelst eines von dem Vortragenden construirten Instrumentes, 2. indem bei der Aufmeisselung des Warzenfortsatzes das Antrum nach vorn erweitert wird, 3. durch vollständige Abtragung der vorderen Wand des Antrums gleichzeitig mit der Wegnahme der unteren und äusseren Wand des "Kuppelraumes".

Discus sion. Katz (Berlin) hatte einige Operationen Küster's mit angesehen, sah dabei auch eine Verletzung des Halbzirkelkanals; $\mathrm{K}$ uster zieht ähnliche Verletzungen nicht in Betracht, er meisselt Alles weg, was cariös ist, und ist nur darauf bedacht, dass der Sinus nicht eröffnet werde.

W alb (Bonn) ist anch der Ansicht, dass man Alles abtragen 
müsse, was die Eiterung hintanzuhalten pflegt; iberhaupt sei das keine chirurgische Behandlung, wo bei den betreffenden Patienten noch Jahre lang nach der Operation Durchspülungen vorgenommen werden müssten.

Wolf (Frankfurt) sah anch einen Fall, wo der Facialis oberAlächlich gelegen war, nebst Caries der betreffenden Partie, wo der Eingriff sicherlich zur Verletzung des Nerven gefithrt hätte.

Hartmann vertritt die Ansicht, dass bei dem chirurgischen Eingriff stets auch auf das G ehör geachtet werden misse.

Barth (Berlin) hält es nicht fuir richtig, dass Chirurgen ohrenärztliche Operationen machen.

$\mathrm{Katz}$ hebt nochmals hervor, dass er stets der Ansicht gewesen sei, dass bei chirurgischen Eingriffen anch das Gehör in Betracht gezogen werden soll; doch muss er zugeben, wie er sich in einem Falle davon iiberzengen konnte, dass die Labyrinthverletzung quoad vitam nicht nachtheilig war.

Prof. Kessel (Jena) glaubt als Otiatriker den Chirurgen gegeniber Stellung nehmen zu muissen, und man düfte es nicht dazu kommen lassen, dass der Chirurg solche Operationen machen soll, die ihm nicht zustehen.

Prof. Kahn (Strassburg) macht darauf aufmerksam, dass ja jedem Otiatriker, der ein grösseres Material hat, schon Fälle vorgekommen seien, die vom Chirurgen direct gesendet wurden.

Prof. Moos (Heidelberg) erwähnt die Gefahr, dass die Chirurgen mit der Zeit das ganze otiatrische Material fortgenommen haben werden.

\section{Sitzung am 20. September 3 Uhr.}

Vorsitzender: Prof. Kessel (Jena).

1. Privatdocent Siebenmann (Basel), Demonstration ron MetallCorrosionspräparaten des Labyrinths.

Nach eingehender Beschreibung der Herstellung von Abgiissen maceríter Schläfenbeine demonstrirt Vortragender 12 Präparate des Felsenbeins und Labyrinths von Erwachsenen und von Nengeborenen. An denselben sind Verlauf der accessorischen Aquaeductuskanale auf Schnecke und Vestibulum, Spongiosa bei Neugeborenen und beim Erwachsenen, und Vertheilung des Acusticus und Facialis deutlich zn sehen. Auch demonstrirte Vortragender zahlreiche, äusserst plastisch gehaltene Zeichnungen, welche die feinen Verhältnisse bei starker Vergrösserung deutlich machen.

Discussion. Barth (Berlin) fragt, welches die beste Methode der Maceration sei.

Siebenmann empfiehlt die gewöhnliche Methode: das Maceriren bei $50-60^{\circ} \mathrm{C}$. mit Kalilauge.

Kessel (Jena) erwähnt, dass einer seiner gewesenen Assistenten, Dr. Götz, sich ebenfalls mit Anfertigung von Corrosionspräparaten beschäftigte; an diesen Präparaten finden sich unter Anderem auch die pneumatischen Räume vor. Eigentlich giebt es gar keine solche. Denn Redner fand an Durchschnitten, die ex an decaleinirten Felsen- 
beinen machte, die Räume mit Membranen, adenoiden Fiaden wad dergleichen Elementen ausgefullt. Auch macht Redner darauf aufmerksam, dass schon die Alten sagten, dass die Rüume mit Wasser gefúllt wären, und wenn dies, buchstablich genommen, auch nicht ganz so ist, wie kommt es dann, dass man von einer Knochenleitngg mit pnenmatischen Räumen spricht.

Tohsen (Frankfurt) erwähnt im Anschluss 2 Fülle, in denen das Verhalten der Knochenleitung gerade im Gegensatz zu dem gefunden wurde, wie es zu erwarten war, und glaubt, dass auch bier die Abnormität der pneumatischen Räume die Ursache gewesen wäre.

2. Prof. Kuhn (Strassburg), a) Demonstration eines Instrumentes zur Entferumg von adenoiden Vegetationen.

Vortragender construirte dasselbe, um die häufge Operation auch fiir den praktischen Arzt zuganglich zu machen. An die Operation selbat stellt Vortragender folgende drei Bedingungen: 1. soll dieselbe möglichst in einer Sitzung gänzlich beendet werden, 2. sollen die exstirpirten Theile sehleunigst heraus befördert werden, und 3. hat der Eingriff so zu geschehen, dass man die $\mathrm{Nar}$ kose erspare. Mit den zangenformigen Instrumenten gelingt das erste nicht. and mit den löffelförmigen Instrumenten wird häufig nicht Alles herausbekommen, da ein Theil zumeist in den Oesophagus geräth; Redner sah in 2 Fällen sogar, dass etwas in die Bronchien gerieth. Das von dem Vortragenden construirte Instrument ist eine Modificirang nacb dem Instrumente von $\mathrm{Schech}$ und wendet er dasselbe bereits seit $5-6$ Monaten an, das sich auch vorzüglich bewährte. Die zu entfernenden Wucherungen werden damit erfasst und duroh das Zusammendrücken der Gefässe mit demselben wird selbst die Blutung aufs Minimale reducirt. Es scheint allen erwähnten Bedingungen zu entsprechen und kann auch vom praktischen Arzt leicht gehandhabt werden.

Discussion. Prof. Walb (Bonn) macht jährlich 300 mal die Operation, chloroformirt nie und gebraucht einen $K$ nnstgriff, durch welchen es ihm gelingt, die Kinder zum Oeffnen des Mundes zu bringen. Es besteht derselbe in dem Beruhren der hinteren Rachenwand; das Dahinkommen geschieht leicht, da sich Ređner für einen Spatel, selbst bei geschlossenem Munde, zwischen den Zahnreihen stets Platz machen kann, und das Kind öffnet dann auch immer den Mund.

Hartmann (Berlin) legt den Sectionsmitgliedern die Schlingenoperation besonders ans Herz, ein Verfahren, das ohne Blutung und in recht eleganter Weise ausgeführt wird. - Kuhn's Instrument seheint ibm den Nachtheil zu besitzen, dass damit der hintere Rand der Choane leicht verletzt werden könnte.

Kuhn betont nochmals, das Instrument nicht so für den Specialisten, als mehr für den praktischen Arat construirt zu haben.

Barth (Berlin) erinnert an die sehon von Trendelenburg empfohlene Methode, das Kind bei Verschluss des Mundes an den Seiten zu drücken, worauf es den Mund offnet, und wean man im Mund ist, dann soll man die Wange zwischen die Zähne driicken, das Kind wird dann gewiss nicht den Mund schliessen. 
Privatdocent Kilian (Freiburg) erwähnt, er hätte viel mit der Hartmann'schen Schlinge operirt, und hat stets mit derselben sein Ziel erreicht.

b) Demonstration eines nenen Accumulators.

Vortragender arbeitet schon seit melireren Jahren mit demselben, der die stärksten galvanokaustischen Schlingen und Messer glithend zu machen vermag. Die Ladung geschieht mittelst Dynamomaschine oder Bunsen-, resp. Chromsäureelement und genïgt eine Füllung immer auf 6 Wochen, und was die Hauptsache ist, der Strom bleibt stets constant. (Derselbe ist bei Schulze und Isenbeck in Strassburg i. E. in verschíedenen Grössen zu haben.)

Discussion. Hedinger (Stuttgart) verlangt einige Aufklihrungen bezüglich der Ladung des Accumulators, die ihm $\mathrm{K}$ uhn giebt.

3. Prof. Walb (Bonn), a) Ueber die Indieationen und Contraindicationen der Luftdonebe bei Behandlung von Mittelohrerkrankungen.

Vortragender bespricht zuerst die acuten, dann die chronischen Erkrankungen des Mittelohres, bei denen so hänfig die Luftdouche irrationell angewendet wird.

Discussion. Prof. Steinbrügge (Giessen) sieht die Perforation des Trommelfells als Folge eines nekrotisirenden Processes an.

Privatdocent Habermann (Prag) war im Stande, an den Rändern der Perforation Staphylokokken nachzuweisen. - Bezüglich des Missbrauches, der mit der Luftdonche getrieben wird, sehliesst er sich dem Vortragenden an und meint, die Behandlung soll sich in acnten Fällen mehr anf Nase und Nasenrachenraum beschränken.

Privatdocent Kilian (Freiburg) beobachtet Fälle, wo nach dem Paukenhöhlenkatarrh, auf Anwenđung der Luftdouche, eine Eiterung sich einstellte.

Vohsen (Frankfurt) will den Schaden der Luftdoucbe nicht überschätzen; man muss allerdings 2 wischen acutem und chronischem Katarrh stets genauen Unterschied machen.

Prof. Knhn (Strassburg) bekräftigt den durch den Vortragenden erwähnten Missbrauch, der sehr oft naheza gefährlich werden kann.

W alb wiinscht auch genauen Unterschied zwischen den leichten und schweren Fällen za machen, die bezüglich der Luftdonche stets abgesondert werden miissen. Bei der acuten Paukenhöhlenentzindung wird durch die Luftdouche die Perforation des Trommelfells schon dadurch beschleunigt, dass man den ohnehin schon vorhandenen Entziindungsdruck noch mehr steigert.

b) Ueber das moderne Specialistentham.

Vortragender rügt deu Missbrauch, der mit der Ohrenheilkunde in neuer Zeit so häufig getrieben wird, den er durch mehrere prägnante Beispiele erläutert.

Discussion. Prof. Moos (Heidelberg) glaubt im Namen Aller zu sprechen, wenn er mit den Auseinandersetzungen des Vortragenden ïbereinstimmt. Nun wäre es aber an der Zeit, auch Etwas zu thun; er sehlägt vor, dahin trachten zu müssen, dass von jedem Mediciner verlangt werde, dass er das otiatrische Colleg belege.

Hartmann (Berlin) glaubt nicht viel durch die Facultäten er- 
reiehen zu können; es müssten die âztlichen Vereine darum ersucht werden, sie mögen alle Diejenigen für Curpfuscher erklären, die sich fuir Specialisten ausgeben und nicht wirklich sind. Bezüglich des Antrages von Moos glaubt Hartmann, dass der Student in der Ohrenbeilkunde examinirt werden sollte.

Thiry (Freiburg) ist derselben Ansicht; denn wenn nicht gleich viel verlangt wird, dann bleibt es mit der Ohrenheilkunde beim Alten; es genü̈gt nicht, dass der Student blos ausweise, dass er das Colleg belegt hat, sondern or soll zugleich auch einer Prüfung unterzogen werden.

Prof. Kahn (Strassburg) beantragt, die Section möge eine Commission ernennen, die dann die gehörigen Schritte einzuleiten hat. $\left.{ }^{1}\right)$

4. Szenes (Budapest), Zur Aetiologie der genuinen otitis media acuta.

Auf Anregung Prof. Zaufal's in Prag suchte Vortragender den Parallelismus der genuinen $O t i t i s$ nd $P$ ne umonie nachatweisen; es geschah dies auf Grund der klinischen Journale $\mathrm{Z}$ a ufal's, die während 15 Jahren ein Material von 18128 Ohrenkranken umfassen, von denen $1205(=6,65$ Proc.s an acuter Otitis litten. Mit diesen verglich Vortragender die 28676 Falle der internen Klinik Prof: Pribram's, die aus denselben Jaluren datiren, unter denen die Pnelimonie sich blos $1165 \mathrm{mal}(=4,06$ Proc.) vorfand.

Nur viermal zeigten beide Erkrankungen einen ganz gleichen Procentsatz (in den Jahren 1874, 1875, 1883 u. 1884), vier andere Male (in den Jahren 1876, 1878, 1879 u. 1886) war derselbe nahezu gleich, 8 mal aber differirte er und zwar in der Weise, dass die Otitis stets einen grösseren Procentsatz machte.

Vortragender scheidet nach der Eintheilung Weichselbaum's die secundären Formen von den primären aus und zieht die ferneren Schlüsse der geringen Zahl der ersteren wegen immer nur ans den letzteren.

Bezüglich des Geschleehts war die Otitis bei $748(=66,8$ Proc.) männlichen und $372(=33,2$ Proc.) weiblichen Kranken gefunden, die Pneumoniefalle bezogen sich auf $663(=59,5$ Proc.) Männer und 451 (= 40,5 Proc.) Weiber. - Abweichender war der sitz beider Erkrankungen, indem die Otitis in 44,46 Proc. der Fälle rechterseits, in 45 Proc. linkerseits und nur in 10,54 Proc. beiderseits bestanden hatte; die Pneumonie aber tritt bekanntlichermaassen in etwas mehr als der Hälfte der Falle in der rechten Lunge allein, in 33 Proc. der Fälle in der linken und in 15 Proc. in beiden Lungenflügeln auf.

Schliesslich verglich Vortragender das Auftreten beider Erkrankungen, indem der Beginn, resp. die Zeit in Betracht gezogen wurde, in welcher sich die ersten Symptome kundgegeben. Die Fälle wurden den einzelnen Monaten gemäss geordnet und stellte es sicl

1) Dieselbe wurde in der letzten Sitzung aus den Herren Prof. Moos (Heidelberg), Prof. Kubn (Strassburg), Prof. Kessel (Jena), Prof. Wagenhänser (Tübingen), Prof. Walb (Bonn) und Dr. Hartmann (Berlin) zusammengesetzt. Ref. 
durch die tabellarische Zusammenstellung bald heraus, dass, jedes Jahr separatim genommen, in den einzelnen Monaten ein und derselbe Jahreszeit of ganz verschiedene Zahlen sowohl beziglich der einen, als auch bezüglich der anderen Affection aufweist. Insgesammt aber den Procentsatz beider im Durchschnitt genommen, zeigten dieselben blos in 4 Monaten (März, October, November und December) einen grösseren Unterschied, in den ubrigen Monaten warea aber beide Affectionen mehr oder weniger ähulich, oft sogar gan gle ich häufig aufgetreten. (Sammtliche Schliusse wurden an entworfenen Curven demonstrirt.)

Endlich glaubt Vortragender beim Vergleiche beider Erkrankungen immer mehr Aehnlichkeit zwischen Otitis und Pneumonie gefunden zu haben, und die Uuterschiede scheinen blos durch aceidentelle Factoren bedingt zu sein.

\section{Sitzung um 21. September 9 Uhr. \\ Vorsitzender: Prof. Waib (Bonn).}

1. Prof. $\mathrm{Sehwalbe} \mathrm{(Strassburg),} \mathrm{Inwiefern} \mathrm{ist} \mathrm{die} \mathrm{menschliche}$ Ohrmuschel ein rudimenthires 0rgan?

Vortragender geht in seinen Erläuterungen vom rein mor pho* logischen Standpunkte aus und kommt zu dem Resultat, dass nicht die ganze Ohrmuschel, wohl aber ein grosser Theil derselben, nämlich der Anthelix (mit Ausnahme des Crus inferius) und der dem oberen und hinteren Helix entsprechende Abschnitt als verkümmert anzusehen ist, durch Rückbildung ans dem grossen Ohrlöfel der mit langen beweglichen Ohren versehenen Säugethiere entstanden; bei dieser Gelegenheit findet die Anthelixbildung eine genane Besprechung.

Rückgebildet ist ferner der Gehörgangsknorpel, insofern er urspruinglich aus drei getrennten Stücken bestand, die beim Menschen derart verwachsen sind, dass die Incisurae Santorini als Reste ursprünglicher Trennung erhalten blieben. - Die Spina helicis ist ebenfalls eine rudimentäre Bildung; sie entspricht dem Scutulum $=$ Knorpel der Ungulaten, Carnivoren u. s. w.

Zum Schluss erörtert noch Vortragender die Frage, inwiefern die Ohrmuskeln als rilckgebildet angesehen werden müssen.

Discussion. Prof. Kessel (Jena) spricht im Anschluss an die Entwicklungsgeschichte der Ohrmuschel itber die Fun ction derselben. Im Wesentlichen entspricht dieselbe einem Schema von Orgelpfeifen; die Vertiefungen an der Muschel müssen als Resonatoren angesehen werden. - Ohrmuschel und Gehörgang bilden ein zusammenhängendes System, dasselbe ist beweglich und auch die grossen Ohrmuskeln dienen zu Zwecken von Richtungsbestimmungen; an der Muschel sind Resonatoren für die Geräusche, und die Ohrmuskeln sind an den wirksamen Punkten für die Resonatoren angebracht. Bezüglich der Bewegungen der Muschel behauptet Redner, dass manche Mensehen dieselbe mit der Galea aponeurotica bewegen.

\section{Barth (Berlin), Beitrag zar Anatomie der Schnecke.}


An der Hand vorgelegter Photographien und Zelchnugen macht Vortragender einige Mittheilungen tiber die Membrana tectoria, and zwar sollen sich dieselben erstrecken auf ihren Ban, ihre Form, ihre Lage und ihren Einfluss auf die Theile, mit welchen sie zu* nächst in Verbindung steht. - In radiäror Richtung besteht die Membran ans drei Zonen. Die bekannte Streifung der inneren und mittleren Zone verläuft im innersten Theile spiralig um den Modiolus herum; weiter nach aussen wendet sie allmählich immer mehr von dieser Richtung ab, um sich mehr der einer Tangente za nähern; in der mittleren Zone kommt sie schliesslich der Richtung eines Radius näher, als der eimer Tangente. In der inneren Zone liegen die Streifen annähernd parallel zu einander und in der Membran selbst. Es gelingt hier selten, durch Zerzupfen die Streifen von einander zu isoliren; wo es gelingt, reisst aber stets die ganze Membran mit durch. In der mittleren Zone ist die Streifung wesentlich deutlicher und besteht ans einzelnen sehr widerstandsfähigen Fibrillen, welche sich durch Zerzupfen leicht isoliren lassen. Ihre innere Befestigung reisst dabei selten los, die äussere sehr leicht, so dass der änssere Rand der zweiten Zone manchmal wie aufgefranst erscheint, wean die dritte Zone völlig abgerissen ist. In dieser Zone kann die Continuität der Membran erhalten bleiben, trotzdem Fibrillen losgerissen sind. An der Membrana tectoria, die frisch der Schnecke einer 40 jährigen Frau entnommen und mit Methylenblau gefärbt war, fand Vortragender nach dem Zerzupfen eine Stelle, an welcher die Fibrillen beseitigt waren. Die dadurch entstandene Liicke bildete aber kein Loeh, sondern wurde durch ein gleichmässig gefärbtes Gewebe ausgefüllt. An Schnitten, welche in den verschiedensten Richtungen durch die Membran gehen, sieht man in der zweiten Zone durchschnittene Fibrillen auf der oberen und unteren Fläche; zwisehen beiden sieht man keine Durchsehnitte von Fasern, sondern einen leeren Raum, oder richtiger die continuirlich in ihrer Richtung verlaufenden Fasern der oberen und unteren Fläche. Hieraus sehliesst Vortragender: Die zweite Zone der Membranbesteht aus einer oberen und einer unteren Lage von Fibrillen; zwischen beiden befindet sich eine Schicht homogenen Gewebes. Die scheinbare Krenzung der Fibrillen in Flächenpräparaten in der mittleren Zone ist dadurch zu exklären, dass bei Einstellen der einen Fibrillenlage stets die andere mit durchscheint, dass aber beide Lagen, selbst wenn sie in völlig gleicher Richtung nach aussen ziehen, wegen ibres gebogenen Verlaufs sich perspektivisch nur selten vollständig decken. Der je nach der Einstellung mehr helle oder dunkle Streifen, ungefähr in der Mitte der zweiten Zone, ist, da der radiäre Durchschnitt der Membran an dieser Stelle der Form eines Linsendurchschnittes entspricht, als optische Erscheinung zu erklären. An den äusseren Rand der zweiten Zone setzt sich die dritte an als spiraler Streifen, von welchem weiter nach aussen sich ein unregelmässiges Maschenwerk fortsetzt. Letzteres konnte bisher nur bis an drei Mam schenreihen beobachtet werden. Damit schliesst es aber nicht ab, sondern die die Maschen bildenden feinen Bänder zeigen sich abge- 
rissen und zum Theil ungesehlagen, ein Zeichen, dass die Membran noeh weiter geht und am änsseren Ende irgendwie befestigt sein muss. Aufklärung hierüber gab Vortragendem ein Präparat aus dem Ohre eines ausgewachsenen Kaninchens. Die Schnecke war entkalkt und vor der Einbettung etwas gedrüekt, so dass am radiären Durchschnitt die Lamina spiralis dem Ligamentum spirale wesentlich genähert war. Die Membrana basilaris mit dern Corti'schen Organ war nach unten gedrängt. Die Membrana tectoria hatte sich mit ihrer inneren Zone von der Crista spiralis erhoben und schwebte \$-förmig gebogen frei im Ductus cochlearis. Nach ausen aber fand sieh diedritte Zone verlängert als äuserst feine, stark lichtbrechende, theils mit einander in Verbindung stehende Fasern, welche bis nach der Prominentia spiralis des Ligamentum spirale zogen und sich biex $z$ wischen den Fasern des letzteren verloren.

Nach dieser oberfächlichen Besprechung kommt nun Vortra. gender noch einmal etwas eingehender anf die einzelnen Theile zurück. Der innere Rand dex inneren Zone wird von den meisten Autoren ganz glatt gezeichnet. Er besitzt aber höckerige, lappige und bandartige Fortsätze, die nicht nur vom Rande, sondern oft anch ein Stuick weiter aussen von der Fläche der Membran ausgehen. Auch an Durchschnitten sieht man nicht selten ähnliche Abzweigungen von der unteren Fläche der inneren Zone nach der Crista spiralis zu herabsteigen. Der innere Rand der Membran erscheint an Flächenpräparaten und an Durchschnitten, im Vergleich an dem direct nach aussen angrenzenden Theil, meist etwas verdickt. Die eigen. thümliche netzähnliche Zeichnung, welche oft an Zupfpräparaten auf der unteren Fläche der inneren Zone zu sehen ist, fasst Vortragender nicht als einfache Eindrücke der Zellen und Wiilste der Spiralleiste auf, sie müssten sonst der Form der letzteren mehr gleichen, sondern als eigenes Gebilde. Alle die zuletzt erwähnten Bildungen sieht Vortragender im Wesentlichen als Befestigungsmittel der inneren Zone, in ihrer Lage auf der Crista spiralis, an. Die äusserst dünne innere Zone nimmt nach anssen nur sehr allmählich etwas an Dicke zu, wesentlich schneller dagegen za Beginn der zweiten Zone. Die Gestalt der letzteren möge man sich im Durchschnitt nngefähr wie die Durchschnittsfigur einer Convexlinie vorstellen, obwohl sie in ihrer normalen Lage sicher etwas anders aussieht. Sie geht in gesehwungener Linie über den Sulcus internus und die Stïtzpfeiler linweg, um sich mit ihrem äusseren Theile auf die äusseren Haarzellen herabzusenken. Vortragender glaubt hier eine Verbindung der Membran mit den darnnter liegenden Theilen annehmen zu müssen, kann aber nicht sagen, wie und wo dieselbe statthat. Oft sieht man, wenn die Membran sich abhebt, Fibrillen an dieser Stelle losgerissen, ja letztere sieht auch ganz rauh und uneben aus, was man an anderen Stellen der Membran nie bemerkt. Die dritte Zone steigt nun in der Gegend der äusseren Haarzellen anf die Hensen'schen Stiitzzellen herab, legt sich diesen sowie den Claudius'schen Zellen fest anf. Sie erseheint auf Durchschnitten als schmaler, stark licht- 
brechender Streifen, ist auch auf Flächenpräparaten, trotz ibrer grossen Durchsichtigkeit, nicht selten zu erkennen und erstreckt sich in ihrem äusseren Theile, verhältnissmässig fest mit ihrer Unterlage verbunden, bis auf das Ligamentum spirale. Das Netzwerk derselben ist unregelmässig, besteht aus Bündern und nicht etwa aus wehr oder weniger runden Fasern. $O b$ die dritte Zone bis zu ihrem äusseren Ende netzförmig ist, kann Vortragender nicht sagen. - Ansser den beschriebenen findet man auch Bilder, welche die Ueberzeugung aufdrängen, dass in der dritten Zone, ganz am Anfang derselben noch vor Abgang des Netzwerkes andere, ebenfalls stark liehtbrechende Fasern nach unten gehen, die sich an Zupfpräparaten leicht auf die untere Fläche der mittleren Zone umschlagen. Vielleicht sind dieselben mit den mehrfach herabsteigenden Verbindungsfäden identisch, welche bei Embryonen sehr deutlich zu sehen sind; vielleicht sind sie auch gleichbedentend mit den Fäden, welche man manchmal in der dritten Zone statt des Netzes an Zupfpräparaten vom Randstreifen abgehen sieht, wenn man nicht vorzieht, letztere als Kunstproduct aus dem Netzwerk zu betrachten. - Die Lage, wie sie Vortragender als normale hingestellt hat, findet man an Präparaten selten. Die Membran besitzt eine grosse Neigung, mehr oder weniger, und zwar meist in der Gegend zwischen erster und zweiter Zone nach oben anfzurücken oder sich sogar über einander zu schlagen, so dass die zweite Zone uber die erste zurickgezogen erscheint. Dabei nimmt sie sehr häufig von der Membrana basilaris losgerissene Zellen des Corti'schen Organs mit nach oben, ein Zeichen, dass die Verbindung der Membrana tectoria mit denselben eine sehr lose durchaus nicht sein kann. Ausser dem Aufrichten tritt aber oft auch das Gewebe in die zweite Zone selbst ein, so dass der losgerissene änssere Rand mit einem grösseren oder kleineren Stück der dritten Zone, die sich dann meist nach oben umschlägt, fast bis auf den Rüeken der Membran wandert. Hieraus und aus den perspectivisehen Bildern, welche nicht nur die Schnittw, sondern auch einen Theil der oberen oder unteren Fläche dem Ange darzubieten pflegen, erklärt sich die so mannigfache Gestalt derselben. Fügt man den letzten Angaben noch hinzu, dass die Membran beim Erheben nicht selten die Reissner'sche Membran mitnimmt, so zeigt das von einer gewissen elastischen Kraft, welche derselben in radiärer Richtung inne wohnt. Zum Theil erklärt sich dieselbe wohl, wenn man eine gewisse Längsspannung der in ihrer Längsrichtung sehr elastischen Membran annimmt, die anch entwicklungsgesehichtlich wahrscheinlich ist.

So lange man der bisherigen Anschaung folgte, dass die Membrana tectoria aussen frei endige, lag kein Grund vor, ibr ohne Weiteres eine directe Einwirkung auf das Corti'sche Organ zuzuschreiben. Sobald man aber annimmt, dass die elastische und gespannte Membran in der vorher beschriebenen Weise befestigt ist, muss man darauf achten, wie sich das Corti'sche Organ zu ihr verhält, und man findet, wenn die Membrana tectoria an einem Präparat sich in der vom Vortragenden als normal angegebenen Lage befindet, so ist der innere $S t u t z$ - 
pfeiler gestreckt und in seinem oberen Theile nach aussen gezogen; der äussere erscheint zusammengedriickt und ungefähr in seiner Mitte nach dem Tunnel zu ziemlich stark geknickt. Mit den Stiitzpfeilern sieht man das ganz Corti'sche Organ etwas niedergedrückt und im oberen Theile nach aussen gezogen. Sobald der äussere Theil der Membran sich loslost und nach oben hebt, strecken sich die $\mathrm{Stiltzpfeiler}$ und stehen annähernd wie ein gleichschenkliges Dreieck uber der Membrana basilaris; mit ihnen riickt sich das ganze Cort'sche Organ etwas nach oben. Letzteres wird also nicht nur durch die Stuitzpeiler sammt der Membrana retieularis in seiner bestimmten Lage erhalten, sondern ganz wesentlich auch dureh die Membrana tectoria. Auch physiologiseh kommt der letzteren, nach der Ueberzeugung des Vortragenden, eine weit grössere Bedeutung zu, als man bis jetzt annimmt; er will sich jedoch nicht in Hypothesen verlieren, für welche obige Darstellung ein weites Feld bietet. Eine eingehendere Bearbeitung der angeregten Fragen nebst einigen im Vorhergehenden nicht bertihrten Pankten wird Vortragender noch folgen lassen.

Discussion. Katz (Berlin) glaubt, die Membrana tectoria nur als cuticuläres Gebilde ansehen zu mïssen.

3. Bronner (Bradford), Ueber Menthol und Eucalyptus und deren locale Anwendung bei Affectionen des Mittelohres.

Vortragender wendet seit 2 Jahren Menthol und Eucalyptus bei Affectionen des Mittelohres und der Eustachi'schen Röhre an. Die günstigen Fälle scheinen recidivirender Katarh und Sklerose in den Anfangsstadien zu sein. Ist starke Schwellung der Schleim. haut der Eustachi'schen Röhe vorhanden, so verordnet Vortragender ein Schnupfpulver von Borsäure und Menthol (2 Proc.) und bläst ausserdem auch noch Mentholdämpfe durch den Katheter. Er benutzt eine Kapsel, ähnlich der Zaufal'schen, die mit Stïcken von Bimsstein gefüllt ist. Die Menthollösung (in Olivenöl oder Alkohol, 2proc.) wird auf den Bimsstein gegossen, die Kapsel an den Lncaeschen Doppelballon befestigt und die Dämpfe dann langsam in das Mittelohr geblasen. Bei starker Affection des Mittelohres werden auch noch einige Tropfen Eucalyptusöl auf den Bimsstein gegossen, bei starker Secretion aber Terpentin- oder Cubebenöl. Stets muss aber für đas gehörige Freisein der Eustachi'schen Röhre gesorgt sein. $\mathrm{Zu}$ diesem $\mathrm{Zwecke}$ benutzt Vortragender dicke konische Bougies aus Celluloid, deren Ende in Mentholöl getaucht wird. Die giünstigen Erfolge führt Vortragender theilweise darauf zurïck, dass er die Dämpfe längere Zeit hindurch auf die Schleimbäute einwirken lässt.

Bei der Behandlung von Otorrhöen scheint das Menthol von keiner besonderen Wirkung zu sein. - Auch bei der Behandlung von Furunculose des äusseren Gehörgangs konnte Vortragender keine gïnstigen Erfolge mit Menthol erzielen und somit kana er die Angaben Cholewa's ') durchaus nicht bestätigen.

1) Therap. Monatshefte, 1589. Nr. 6.

Arehiv f, Ourenheilkunde. XIIX. Bd. 
Discussion. Barth (Berlin) erwähnt, er hätte ein Instrument zur leichteren Application des Mentholöls construirt.

Hartmann (Berlin) erwähnt, dass Cholewa's Aufsatz eigentlich ans seinem Ambulatorium stamme, doch theilt er nicht ganz die $\Delta n=$ sicht bezugglich der giinstigen Erfolge des Mentbols und bat anch Cholewa, seinen Namen gar nicht zu erwähnen.

Szenes (Budapest) hatte, im Verein mit dem klinischen Assistenten Dr. Anton in Prag, das Menthol in mehreren Fällen von Furunculose des äusseren Gehörgangs versucht und will demselben zwar nicht alle guten Eigensehaften absprechen, doeh darf es keineswegs als Antifuruneulosum hingestellt werden; es vermochte blos in 3 Fällen (von 12) einen schnelleren Ablauf der Erscheinungen zu verursachen, ruft jedoch nur zu oft selbst ein lästiges Brennen hervor, vermochte anch nicht Recidive zu verhüten, und wenn man trotz Alledem das Menthol so warm empfiehlt, wie es Oholewa thut, dann werden auch die praktischen Aerzte dazu verleitet, es anzuwenden, und wenn es auch diese im Stiche lassen wird, was voraussichtlich zu erwarten ist, kann das nur zum Misscredit unserer Specialität beitragen.

4. Privatacent Habermann (Prag), a) Ueber Tarbbeit der Kesselsehmiede.

Vortragender hatte Gelegenheit, die Gehörorgane eines 75 jährigen Mannes untersuchen zu können, der infolge einer mehr als 20jahrigen Beschäftigung in einem Kupferhammer taub geworden war. Derselbe irrte später als Bettler herum, bis er eines Tages über ein Bahngeleise ging und von dem heranfahrenden Eisenbahnzug getödtet wurde. - In beiden Gehörorganen fand sich ein Fehlen der Nerven der Lamina spiralis und ein Schwund der Ganglienzellen des Rosenthal'schen Kanals, in der basalen Windung von der vorderen Umbiegungsstelle angefangen bis zum Ende, und setzte sich dieser Schwund der Nerven noch bis in den Stamm des Gehörnerven fort. Das Cortische Organ, das in der iibrigen Schnecke schön erhalten war, fehlte an der erwähnten Stelle ganz oder waren nur indifferirbare Reste desselben vorhanden, die Membrana basilaris mit Pflasterepithel bedeckt, die Nerven in der übrigen Schnecke in ibrer Stärke anf die Hälfte reducirt. Beiđe Steigbügelfussplatten waren stark nach aussen gerückt, wie es bei starker Contraction des Stapedius geschieht, und fuihrt Vortragender dies anf die Function des Stapedius zurück, als Schutzvorrichtung gegen das Einwirken starken Schalles auf das Laby. rinth zu wirken. Die Atrophie der Nerven entstand in der Weise, dass durch die langdauernde Einwirkung des starken Schalles die Endausbreitungen der Schneckennerven gelähmt wurden und diese Lähmung dann zu Schwund des ausser Function gesetzten Corti'sehen Organs und zur anfsteigenden Atrophie der Nerven fuihrte. Dass das Fehlen der Nerven auf das Ende der Schneckenbasis beschränkt war, erklärt sich daraus, dass bei der Arbeit vorwiegend Schall von hohem Toncharakter einwirkte, und stimmt dies anch mit den bis= herigen klinischen Erfahrungen, nach denen Abschwächung der Knochenleitung und Taubheit für hohe Töne für diese Erkrankung cha- 
rakteristisch sind. Vortragender konnte dies durch eigene Unter: suchungen einer Anzahl von Kesselschmieden bestätigen.

Discussion. Prof. Kessel (Jena) macht darauf aufmerksam, dass die Gehörknöchelchen an Präparaten freigelegt werden können, so dass man die hervorgerufenen Bewegungen anch mittelst Mikroskops prüfen könne.

b) Zur Entstehung des Cholesteatoms des Mittelohres mit Demonstration mikroskopischer Praparate.

Vortragender hat schon friher I), gestiitzt auf die genaue histologische Untersuchung eines Falles, ferner anf langjährige klinische Erfabrung und anf eine Anzahl ähnlicher Fälle in der Literatur, die Behauptung aufgestellt, dass die Mehrzahl der Cholesteatome des Mittelohres in der Weise entsteht, dass die E pidermis des äusseren Gehörgangs, resp. des Trommelfells bei chronisehem Verlauf einer Mittelohrentziindung in das Mittelobr hineinwächst und hier bei der Fortdauer der Entzündung zu vermehrter Abstossung der Hornschicht und zur Ansamming dieser Masse der Hornschicht in Form der Perlgeschwulst fuhrt. Seitdem hatte Vortragender Gelegenheit, die Richtigkeit dieser Behauptung durch die Untersuchung zweier weiterer Falle von Cholesteatom des Mittelohres bestatigt zu seben, und theilt einen dieser Fälle, bei dem sich zwei getrennte Perlgeschwillste in einem Ohre fanden, ausfuhrlich mit. Das eine der Cholesteatome war von kngelförmiger Gestalt und füllte die Paukenhöhle, das Trommelfell stark nach aussen drängend, aus. Es war aussen von Schleimhant bekleidet, innen von Epidermis und hing einerseits mit dem Promontorinm zusammen, mit dem es verwachsen war, andererseits mit dem Trommelfell, in welchem durch eine kleine Perforation in der centralen Partie die Epidermis des Trommelfells uber den Perforationsrand lineingewachsen war und dort das Cholesteatom innes anskleidete. Die Membrana propria war nur eine kurze Strecke weit in der Wand des Cholesteatoms an verfolgen und die Schleimhantschicht des Trommelfells bildete in ihrer Fortsetzung die äussere Bekleidung das Cholesteatoms in der Paukenhöhle. Dieses Cholesteatom dürfte in der Weise entstanden sein, dass sich im Verlaufe der Mittelohrentzündung eine mit dem Promontorium verwachsene Trommelfellnarbe bildete, die sich mit dem Abheben des Trommelfells sackförmig auszog und in der sich nun die bei der fortdanernden Entzindung lehhafter sich abstossenden Hornschichten ansammelten und so den Sack immer mehr vergrösserten.

Das zweite Cholesteatom kleidete das Antrum mastoideum und den binteren oberen Theil der Paukenhöhle ans und hing direct mit der Epidermis des äusseren Gehörgange durch den hinteren Theil der Shrapnell'sehen Membran zusammen.

Discussion. Prof. Walb (Bonn) schliesst sich der Ansicht des Vortragenden über die Entstebrng des Cholesteatoms an.

Prof. Kuhn (Strassburg) macht auf die Fäle aufmerksam, wo

1) Dieses Archiv. Bd. XXVII. 
Cholesteatome in unversehrten Warzenfortsatzhöhlen entstehen, und glanbt das Cholesteatom in manchen Fällen als primären Tumor ansehen zu müssen.

Barth (Berlin) betrachtet das Cholesteatom als durch Degeneration der Schleimhaut verursacht, indem eine Anlagerung von verhornten Epidermisschichten stattindet.

Habermann macht auf Billroth's Ansspruch anfmerksam, der das Cholesteatom als fötalen Ursprungs deutet.

Prof. Kessel (Jena) ist der Ansicht, dass das Cholesteatom sonderbaren Ursprung haben muss, der oft nicht leicht $z \mathfrak{u}$ erklären wäre.

Prof. Ste in brügg e (Giessen) meint, dass zum Entstehen eines Cholesteatoms zwei Factoren nöthig wären: das Hereinwachsen der Epidermisschicht und ansserdem noch eine Dermatitis.

\section{Sitzung am 21. September $3 \mathrm{Uhr}$.}

Vorsitzender: Dr. A. Hartmann (Berlin).

1. Vohsen (Frankfurt), Zur Therapie der Mundathmung mit Demonstration.

Nach operativer Beseitigung der die Nasenathmung verhindernden Ursachen ist bekanntlich die Mundathmung in vielen Fällen noch nicht aufgehoben. Bei so manchen Mundathmern sind Hindernisse in der Nase und im Nasenrachenraum nicht vorbanden und der falsche Athmungstypus kann nur als Folge von fritheren pathologisehen $\mathrm{Za}$ ständen aufgefasst werden, wie eine zu grosse Rachentonsille, consecutive Nasenkatarrhe, nach deren Schwinden die Gewohnheit des Mundathmens zurickbleibt.

Zur Hebung der Mundathmung bei durchgängiger Nase sind mehrfach Apparate angegeben worden. Die einen verschliessen die Lippen, die anderen drängen den Unterkiefer gegen den Oberkiefer. - Der erste war der von Guye als "Contrarespirator" (1874) angegebene Apparat, der in einem mit Seide gefiutterten ovalen Stuck Wachstuch besteht, das uber den Mund gebunden wird. - Gardon fertigte einen von Mackenzie-Semon erwähnten Celluloidapparat an, der zwisehen Lippen und Zähnen getragen wird. Bloch erwähnt einen weiteren Apparat der internationalen Verbandstofffabrik in Schaffhausen, der im Wesentlichen aus einem schalenartigen dünnen Stückchen Hartgummi oder einer ähnlichen Masse besteht, sich den Zähnen dicht anlegt und in der That seinen Zweck erfüllt, indem die Mundathmung durch das Tragen des Apparates in hohem Grade erschwert wird. Gegen die beiden letzten Apparate richtet sich aber der wohlberechtigte Einwurf Bloch's, dass man Anstand nehmen müsse, ihn Kindern Nachts im Munde zu belassen, da die Gefahr des Hineingerathens in den Larynx oder die speiseröhre nicht ausgeschlossen ersoheint. - Delstanche schlägt vor, durch ein um Kinn und Scheitel gelegtes Tuch den Mund zu verschliessen. Das hat wohl mancher Mundathmer sehon von selbst versucht und dabei erfahren, dass ein solcher Verband ebensowenig festhält, wie der Guye'sche 
Apparat. Der Mundathmer, dem sein gewohnter Athmungstypus unmöglich gemacht wird, schläft unruhig und nur der sehr fest sitzende Apparat wird seinen unwillkürlichen Entfernungsversuchen Wider. stand leisten.

Ein Apparat, der das Mundathmen unmöglich machen soll, muss nicht nur den Unterkiefer gegen den Oberkiefer pressen, sondern auch die Lippen verschliessen. Ist blos Ersteres der Fall, so klaffen bei sehr vielen Mundathmern die Lippen und es kann durch eventuell vorbandene Zahnlïcken, oder die natïrliche Lücke zwischen oberer und unterer Zahnreihe respirirt werden. Versehliesst man hingegen nur die Lippen, so wird damit noch nicht dem Unterkiefer die Lage gegeben, welche die Zunge nöthigt, dem Ganmen fest anzuliegen. Damit wird schon ein Theil der hygienischen Vortheile der Nasenathmung aufgehoben. Weiter aber behält infolge der Gewohnheit der Unterkiefer die Tendenz, zu fallen, und der Mundathmer verfällt viel leichter wieder in den früheren Athmungstypus.

Der Apparat, den Vortragender vorzeigt, erfüllt die Anforderungen, welche wir an einen Mundschliesser stellen müssen, indem er erstens festsitzt, zweitens den Leidenden nicht belästigt (die Kinder gewöhnen sich nach 1-2 Nächten schon daran), und drittens verschliesst er die Lippen und presst zugleich den Unterkiefer gegen den Oberkiefer.

Der Apparat besteht aus zwei Gummiplatten von verschiedener Grösse nnd ovaler Form. Sie sind am Rand von einem luftgefüllten Schlauch umgeben. In die grössere reicht das Kinn, die kleinere Platte ist für den Mund bestimmt. Das Band, das die Kinnplatte hält, theilt sich in der Höhe des oberen Ohrmuschelrandes gabelförmig: und soll die beiden Scheitelbeinhöcker zwischen sich fassen. Unterhalb des Ohres laufen durch zwei Desen die Bänder, welche die Mundplatte tragen. Dieselben werden in der Nackengegend durch eine Schnalle verbunden.

Vortragender glaubt, der Gu ye'sche Name Contrarespirator sei für einen solchen Apparat keine glïckliche Bildung; er soll ja das Respiriren nicht hindern. Der Apparat könnte zweckentsprechender Anoralrespirator genannt werden, das bezeichnet am besten die Wirkung, die er hervorbringen soll. (Der Apparat wird in zwei Grössen von der Gummiwaarenfabrik von Weil, Frankfurt a. M., Töngesgasse, zum Preis von 5 Mark gefertigt.)

2. Prof. $K$ es sel (Jena), Demonstration von elektrischen Beleuehtungsapparaten fiir das Mittelohr und den Nasenrachenraum.

Zuförderst besprieht Vortragender eingehend die versehiedenen Beleuchtungsmethoden und deren praktischen Werth von der Erfindung des Ohrenspiegels bis zum heutigen Tag und komm abliesslich auf das elektrische Licht zu sprechen, um dana dis duter/ ing construirten Vorrichtungen zu demonstriren, mittederen das botreffende Bild bei starker Beleuchtung men fr $x_{\text {gh }}$ vergrös ert gesehen wird, ein Umstand, der zur Diagndse häuffig wesentlich viel beitragen kann. 
3. Hartmann (Berlin), a) Zur Casuistik der Hogmorshoblenm empyeme.

Vortragender berichtet ïber einen Fall von Empyem der Highmorshöhle, der dadurch besonderes Interesse beansprucht, dass der Patient im ersten Stadium der Erkrankung den Verdacht des Vor* handenseins einer Lungenaffection erweckte. Derselbe hatte nämlich ein halbes Jahr hindurch stets Morgens mit Blut vermischten Auswurf, zu dem sich später geringer Ausfluss tibelriechenden Eiters gesellte. Es ergab sich nun, dass das Auftreten der Blutungen genau zusammenfiel mit dem Plombiren eines Zahnes. Nach Extraction desselben und Anbohren der Highmorshöhle strömte ibelriechender Eiter aus der Nase. Schon nach 3 Ausspilnngen war vollständige Heilnng erzielt.

Vortragender erwähnt sodann zwei weitere Fälle von Highmorshöhlenempyem, in welchen es ihm gelang durch 3, resp. 4 Ausspiilungen der Höhle vom mittleren $\mathrm{N}$ asengang aus mit der von ihm früher empfohlenen Röhre vollständige und dauernde Heilung zu erzielen.

b) Demonstration eines nenen Instruments.

Vortragender demonstrirt einen Zerstä abungsapparat für die Application von Cocainlösung in Nase, Nasenrachenraum und in den Kehlkopf. Der Apparat ist an dem als Handgriff dienenden, die Flüssigkeit enthaltenden Glase mit einer Unterbrechungsvorrichtung versehen, durch welche es ermöglicht ist, beliebig kleine Mengen der Lösung zur Anwendung zu bringen.

Discussion. Bloch (Freiburg) hat ein ahnliches Instrument construirt, das kleine Enterschiede hat, die er angiebt.

4. Ein längerer Aufsatz von Prof. Politzer (Wien), Beiträge zur Pathologie und pathologischen Anatomie des Gehörorgans, ebenso der Anfsatz von Treitel (Berlin), Ohrenkrankbiten bei Tabes, die eingesendet wurden, sind nicht verlesen worden; blos das zu Prof. Politzer's Aufsatz gehörende Präparat warde demonstrirt.

Nachdem der Vorsitzende dern Einfuihrenden Prof. Moos den Dank der Section ausgesprochen, wurde auch den Vorsitzenden der Sitzungen für die umsichtige Leitung der Discussionen und den Schriftfuihrern für ihr mulhsames Amt gedankt.

In die Präsenzliste hatten sich eingetragen:

Dr. Albers (Saarlouis), Dr. A ppert (Karlsruhe), Dr. Barth (Berlin), Dr. Becker (Dresden), Dr. Beckmann (Heidelberg), Dr. Bloch (Freiburg), Dr. Bö ck (Magdeburg), Dr. Bronner (Bradford), Dr. Buss (Darmstadt), Dr. Cimbal (Neisse), Dr. Döring (Ems), Dr. Ferrer(San Franziseo), Dr. Friederichs (Elberfeld), Dr. Friedländer (Bad Nauheim), Privatdoeent Gottstein (Breslau), Privatdocent Habermann (Prag). Dr. Hartmann (Berlin;, Dr. Hecke (Breslan), Dr. He dinger (Stuttgart), Dr. H erbordt (Frankfurta. M.), Privatdocent Heymann (Leipzig), Dr. Jacoby (Magdeburg), Dr. Kassnitz (Karlsruhe), Dr. Katz (Berlin), Dr. Katzenberger (Baden-Baden), Dr. Keimer (Düsseldorf), Prof. Kessel (Jena), Pri- 
vatdocent Kilian (Freiburg i.B.), Dr. Kilian (Worms), Dr. Koerner (Frankfurt a. M.), Prof. K uln (Strassburg), Privatdocent Lemeke (Rostoek), Dr. Lommel (Homburg), Dr. Lenz (Bretten), Dr. Lin kenheld (Wiesbaden), Dr. Mayer (Mainz), Dr. Messer (Mannheim), Prof. Moos (Heidelberg), Dr. Proebsting (Wiesbaden), Dr. Roller (Trier), Dr. Rothschild (Wallrok), Dr. Sacki (Pforzheim), Dr. Schllferowitsch (Heidelberg), Prof. Schwalbe (Strassburg), Privatdocent Siebenmann (Basel), Dr. Stark (Cincinnati), Prof. Steínbrügge (Giessen), Dr. Stöhr (Birkenau), Dr. Strazza (Genua), Dr. Szenes (Budapest), Dr. Thiry (Freiburg i. B.), Dr. Truckenbrod (Hamburg), Dr. Usinger (Bensheim), Dr. Vohsen (Frankfurt a. M.), Prof. Wagenhä n ser (Tübingen), Dr. Wähe (Kirchardt), Prof. Wa l b (Bonn), Dr. Weydner (Neckar-Steinach) und Dr. Wolf (Frankfurt a. M.). 